\title{
Research on Simulation and Collision Check in Three-Dimensional Inspection Planning
}

\author{
Guijiang Duan ${ }^{1, a}$, Shuai Gao ${ }^{2, b}$ \\ ${ }^{1}$ School of Mechanical Engineering and Automation, Beihang University, Beijing, 100191, China \\ ${ }^{2}$ School of Mechanical Engineering and Automation, Beihang University, Beijing, 100191, China \\ aemail: gjduan@buaa.edu.cn, bemail:gaoshuai635580552@163.com
}

\section{Keywords: Pro/E; CAIP; Trajectory; Simulation; Collision Check}

\begin{abstract}
Simulation and collision check are the essential function to avoid the potential collision in inspection process based on three-dimensional Computer Aided Inspection Planning (CAIP), especially in the complex parts measuring, which can promote the efficiency of digital measuring equipment such as Coordinate Measuring Machine (CMM). In this paper, the method of generation and visualization of trajectory with the macro command, the monitoring visualization by simulation and the intersection of sweep volume in collision check are discussed and validated though a practical case based on Pro/E.
\end{abstract}

\section{Introduction}

With the development of manufacturing industry, the requirements on the accuracy of products are put forward especially in areas such as aerospace, automotive and mold where not only the structural designs of products are complex, but also the manufacturing processes of parts are difficult. [1] In order to guarantee the quality of products, the application of digital measuring technology is becoming more and more important. [2]

The information of CAIP based on three-dimensional model comes directly from the designs of product, which can generate the measuring program and drive the digital measuring equipment to accomplish the inspection tasks. By means of computer aided method such as the optimization of measuring trajectory and collision check, the measuring equipment can avoid the potential collision before the actual inspection process so as to promote the efficiency and accuracy of measuring.

The current related researches about simulation and collision check based on three-dimensional model, however, mainly cover the single technology or independent modules, rather than the overall consideration in trajectory generation, simulation and collision check. The whole consideration of internal relationships between them can create an optimal solution to the inspection planning.

In this paper, the algorithm of generation and visualization of trajectory with the macro command, the monitoring visualization by creating a continuous "frame" with the algorithm of transformation matrix and the intersection between sweep volume and product in collision check are proposed and discussed based on Pro/E.

\section{Visualization and Generation of Trajectory with Macro Command}

The space curve can be created in many ways in three-dimensional model, and the spline with the advantages of smooth curve, accurate precision and easy modification is widely used. [3] The spline can be controlled to roughly generate the trajectory with the points (measure points and position points) in the key position, and the number and position of points directly affect the shape of trajectory. To make the trajectory accurate and smooth, a certain number of interpolation points are inserted between controlled points by interpolation calculation so as to generate a trajectory with the accuracy and visualization.

For example, the coordinates of starting point Pstart and ending point of Pend are (Xstart, Ystart, Zstart) and (Xend, Yend, Zend). The number of interpolation points is $\mathrm{n}$. The general coordinates of interpolation points $\mathrm{Pi}$ are $(\mathrm{Xi}, \mathrm{Yi}, \mathrm{Zi}) . \mathrm{i}=(1,2, \wedge, \mathrm{n})$. To generate the trajectory between two points, 
the mathematical algorithm of interpolation is as follows:

$$
\left\{\begin{array}{l}
\mathbf{X}_{\mathbf{i}}=\mathbf{X}_{\text {start }}+\mathbf{i} *\left(\mathbf{X}_{\text {end }}-\mathbf{X}_{\text {start }}\right) /(\mathbf{n}+\mathbf{1}) \\
\mathbf{Y}_{\mathbf{i}}=\mathbf{Y}_{\text {start }}+\mathbf{i} *\left(\mathbf{Y}_{\text {end }}-\mathbf{Y}_{\text {start }}\right) /(\mathbf{n}+\mathbf{1}) . \\
\mathbf{Z}_{\mathbf{i}}=\mathbf{Z}_{\text {start }}+\mathbf{i} *\left(\mathbf{Z}_{\text {end }}-\mathbf{Z}_{\text {start }}\right) /(\mathbf{n}+\mathbf{1})
\end{array}\right.
$$

The trajectory realized by mathematical interpolation algorithm is shown in Figure 1.

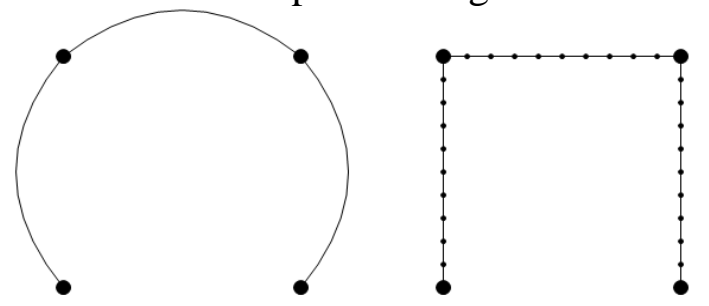

Fig.1. The algorithm of trajectory

The "trail file" is automatically generated in the working directory during Pro/E runtime, which records the users' actions performed in every step of operations, including a lot of macro such as " Command ". The macros can be pushed into the stack and executed immediately through the macro programming so as to realize the function unavailable by Pro/Toolkit. [4]

The model of visualization and generation of trajectory with macros is shown in Figure 2.

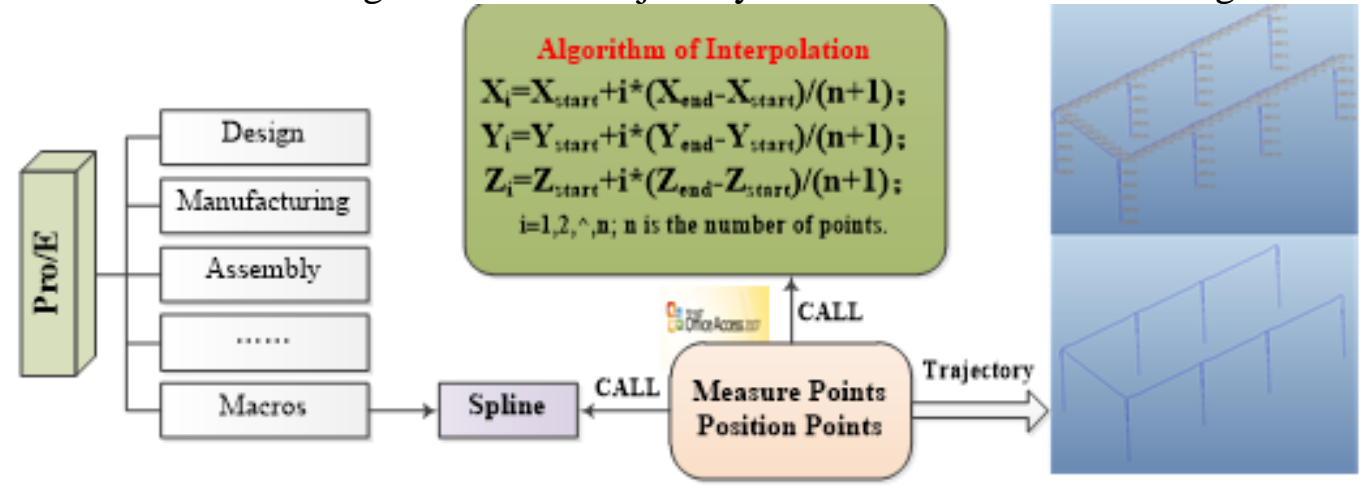

Fig.2. The model of trajectory

\section{Application of Transformation Matrix in Simulation}

Space which is described by the position information of parts, is always occupied by parts in the assembly. [5] The $4 \times 4$ transformation matrix is used to describe the position information of parts in Pro/E. [6] It is shown as follows:

$$
M=\left[\begin{array}{c}
a b c p \\
d e f q \\
h i j r \\
l m n s
\end{array}\right]^{4 \times 4} \text {. And }\left[\begin{array}{l}
a b c \\
d e f \\
h i j
\end{array}\right]^{3 \times 3} \text { which is mainly used for the realization of proportion, }
$$

reflection, rotation and shear of graphics; $[1 \mathrm{~m} \mathrm{n}]^{1 \times 3}$ which is mainly used for the realization of translation; $\left[\begin{array}{l}p \\ q \\ r\end{array}\right]^{3 \times 1}$ which is mainly used for the realization of projection; $[s]^{1 \times 1}$ which is mainly used for the realization of overall proportion.

The simulation in the inspection is realized by matrix $M$ on the basis of measuring coordinate system, but the matrix B is used for model transformation. There is a conversion relationship of matrix A between them. The key step in simulation is to figure out the composite matrix $\mathrm{M}$. It is shown as follows: $\mathrm{M}=\mathrm{A} \mathrm{B}$. The translational motion is the main movement in detection with $\mathrm{CMM}$, so the matrix $\mathrm{B}$ is as follows:

$$
B=\left[\begin{array}{llll}
1 & 0 & 0 & 0 \\
0 & 1 & 0 & 0 \\
0 & 0 & 1 & 0 \\
l & \mathrm{~m} & \mathrm{n} & 1
\end{array}\right]^{4 \times 4} ; \text { and }\left\{\begin{array}{l}
\mathbf{l}=\text { Xstart }+\mathbf{i} *(\text { Xend }- \text { Xstart }) /(\mathbf{n}+\mathbf{1}) \\
\mathbf{m}=\text { Ystart }+\mathbf{i} *(\text { Yend }- \text { Ystart }) /(\mathbf{n}+\mathbf{1}) . \\
\mathbf{n}=\text { Zstart }+\mathbf{i} *(\text { Zend }- \text { Zstart }) /(\mathbf{n}+\mathbf{1})
\end{array}\right.
$$


The matrix $A$ is as follows: $A=\left[\begin{array}{cccc}a & b & c & 0 \\ d & e & f & 0 \\ h & i & j & 0 \\ l & m & n & 1\end{array}\right]^{4 \times 4}$.

The matrix A which has been stored can be read from the database when the measurement coordinate system is built. After the initialization of matrix A, the composite matrix $\mathrm{M}$ can be worked out.

The model of the simulation and monitoring visualization is shown in Figure 3.

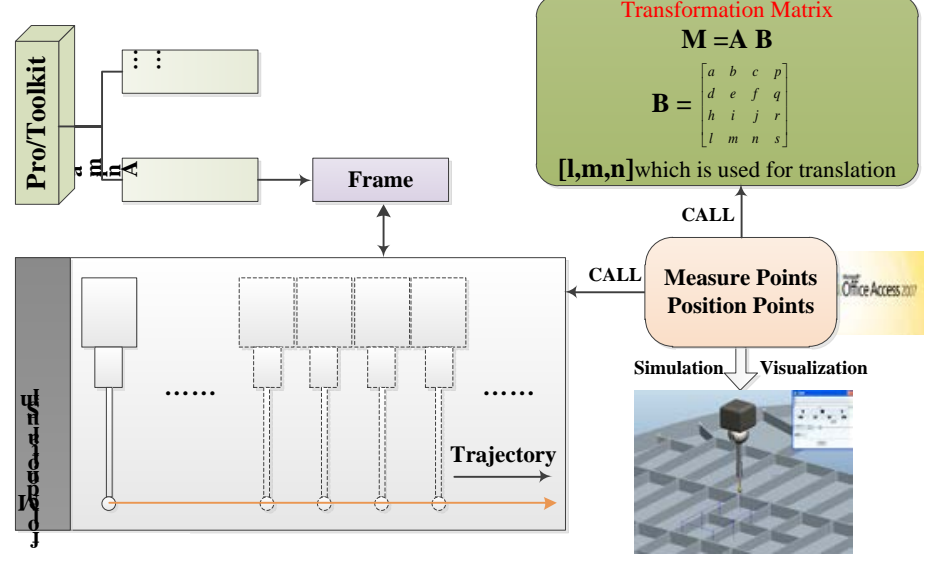

Fig.3. The model of simulation

\section{Realization of Sweeping Intersection in Collision Check}

Compared with the traditional way of interpolation, sweeping intersection in collision check is a more effective and efficient inspection way. [7] By making the model of probe move along the given trajectory, the sweep volume is completely formed. The areas of collision can be gotten between the sweeping volume and the inspected product through the global intersection calculation, which greatly reduces the time of computation and promotes the efficiency of detection.

The formation process of sweeping volume about probe is shown in Figure 4.

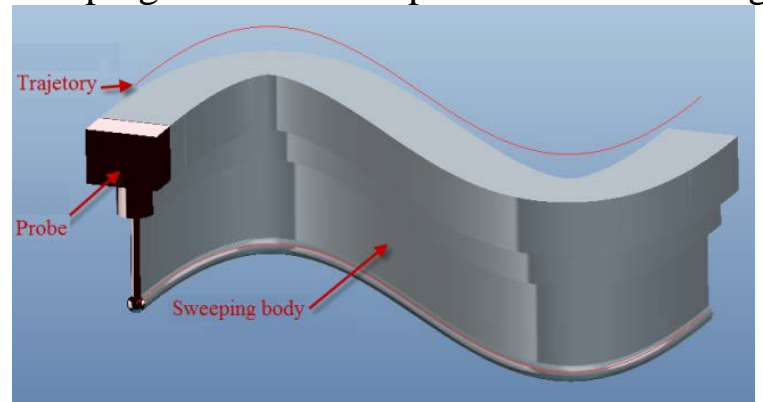

Fig.4. The formation process of sweeping volume

The model of the intersection of sweeping volume is shown in Figure 5.

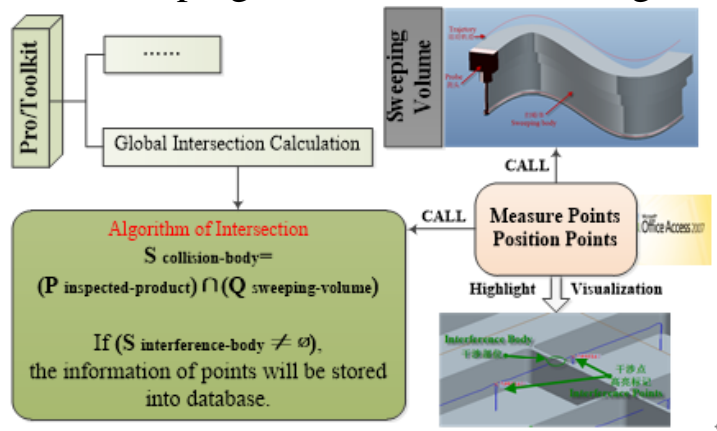

Fig.5. The model of the intersection of sweeping volume 


\section{Case Study}

In trajectory generation, the system reads the information of points about the planned trajectory from the database and automatically generates a number of interpolation points. With the macro commands called in programming, the trajectory is created and displayed in the assembly environment of Pro/E.

In simulation, the system creates the frames of animation to complete the visualization of simulation after the configuration of probe so as to validate the reasonability of the planned trajectory generated before.

In collision check, the system builds the sweeping volume along the planned trajectory with probe and calls the intersection algorithm to verify whether collision does exist in the model. The system will pop up a dialog to show the reason and location of interference with making the point highlight on the three-dimensional model in case of collision.

The inspection results are shown in Figure 6.

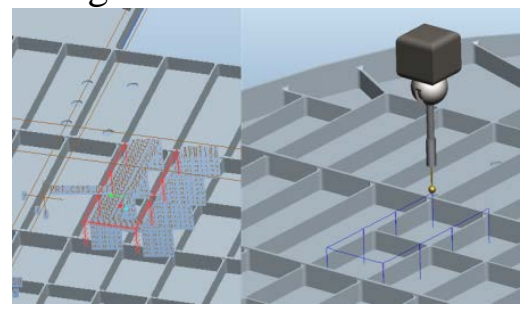

Fig.6. The inspection results

\section{Conclusion}

In this paper, the measuring environment of simulation and collision check is built through the combination of Pro/Toolkit and Visual $\mathrm{C}++$ based on Pro/E. The visualization of trajectory and points causing collision, with the results of inspection planning, ensures the reliability and efficiency of measurement. The models of trajectory visualization, monitoring visualization and intersection of sweeping volume are given in this paper. In addition, the theory is validated through a practical case, making the CMM efficient in inspection.

\section{References}

[1] Cen Rong. Research on Detection Path Planning and Simulation Based on Three-Dimensional Model [D]. Beihang University, Master’s Thesis, 2013.

[2] Wu Bin. The Redevelop of Pro/E Assembly Interference Check [D]. Southeast University, Master's Thesis, 2006.

[3] Zhang Jiang. Research and System Development on Three-Dimensional Inspection Planning for Typical Aircraft Structural Parts [D]. Beihang University, Master’s Thesis, 2009.

[4] PTC. Pro/TOOLKIT Wildfire 5.0 Users Guide [EB]. PTC, 2010.

[5] He Zhangyi. Research of Key Technologies on Intelligent Coordinate Measuring Machine (CMM) [D], 2006.

[6] I. Ainsworth, M. Ristic, D. Brujic. CAD-Based Measurent Path Planning for Free-Form Shapes Using Contact Probes, 2000.

[7] Fiona Zhao, Xun Xu. Computer Aided Inspection Planning. The state of the art, 2009. 